\title{
Development and Application of Self-learning Model for HSM
}

\author{
Ce Wang ${ }^{1, a, *}$, Ziying Liu ${ }^{1, b}$, Lijie Dong ${ }^{2, ~ c}$, Changli Zhang ${ }^{1, d}$, Fengqin Wang ${ }^{1, d}$ \\ ${ }^{1}$ Shougang Research Institute of Technology, Beijing 100043, China \\ ${ }^{2}$ Beijing Shougang CO,LTD, China \\ awangce@shougang.com, bzoonlzy@163.com, cdonglijie2160@sgqg.com, \\ dzhangchangli@shougang.com, êwangfengqin@shougang.com \\ ${ }^{*}$ Ce Wang
}

Keywords: Hot strip rolling, Profile and flatness control, Self-learning.

\begin{abstract}
In recent years, based on maturity of AGC technology, the accuracy of Thickness Control has been improved greatly. The poor profile control is the major factor which restricts the dimensional accuracy. The mathematical model of plate shape-setup is the basis for realizing the process control of strip mill. Because the deviation of the mathematical model, the device and the parameter is inevitable during actual rolling, self-learning is an important mean to reduce the error of the set-up model. On a production line, the self-learning algorithm is not in line with actual production, which results in control chaos. To solve this problem, combined with the actual production process, a new self-learning algorithm has been developed, which considers operator revision, unstable error interference and actual parameters optimization. New algorithms has been applied, it has solved the phenomenon of self-learning disorder, improved the hit rate of profile greatly, and improved the quality of the production.
\end{abstract}

\section{Introduction}

In recent years, many scholars have carried out a large amount of research on the process control model of hot strip rolling, and developed the relevant mathematical model and control system, and made great progress. Hot rolling control system is also becoming more perfect, in the control function, mainly including temperature control, thickness control, width control, shape control, tissue control and mechanical performance control, etc. The control system includes basic automation control, process control, production management control and information management control [1].

Profile is an important control index in the process of hot strip rolling. An excellent profile control strategy of the hot rolling mill is the prerequisite which ensuring the effect of profile control ${ }^{[2]}$. In this paper, a new profile self-learning algorithm has been developed for the cause of self-learning disorder, which considers operator revision, unstable error interference and actual parameters optimization. New algorithms has been applied, it has solved the phenomenon of self-learning disorder, improved the hit rate of profile greatly, and improved the quality of the production ${ }^{[3]}$.

\section{Main Problem}

\subsection{Overview}

Since January 2017, the level of crown control has dropped dramatically. Combined the relevant production data and found that the convexity short-term self-learning model is in disorder, as shown in Figure 1. The problem is mainly manifested at that learning the opposite situation. The setup value is larger than the value which resulting in positive deviation of the palte profile, leading to the accuracy of profile control is worse. 


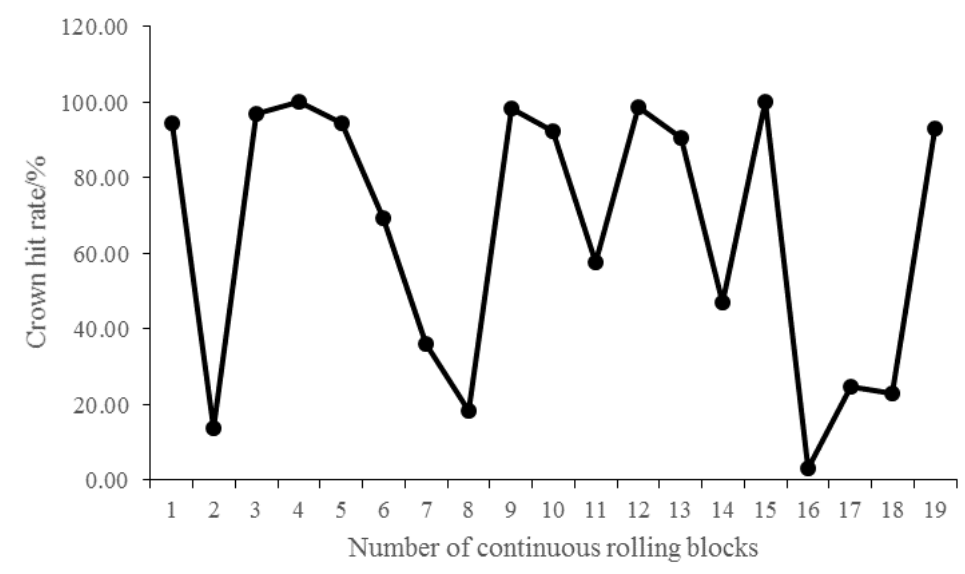

Fig. 1: Examples of Self - learning Disorder

\subsection{Analysis}

The profile setup is calculated based on the data of finish rolling model. The Fundamental Relationship of the shape setup algorithm says that the strip thickness profile at the exit of each individual stand must equal the roll bite contour across the strip width at the roll bite exit. The roll bite profile at the roll bite exit is a superposition of

- the initial work roll crown (ground camber for zero shifting position),

- the work roll bending contour for zero bending force,

- the work roll contour due to flattening (at the strip - work roll interface),

- the thermal and wear crowns of the work rolls,

- the 'actuator crown' resulting from profile and flatness control actuators such as bending

forces and CVC-shifting.

The shape setup algorithm calculate the bending force and channeling value of each frame, and send the setting to the Level-I, which mainly to ensure that the outline of the strip head. With the continuous strip rolling, the thermal and wear crowns of the rolls, rolling force, bending force and the tension are different from the value used in the shape setup caculation, which is bound to affect the outline of the strip, espescially the outline of the body and the tail, so we added the shape self-learning function ${ }^{[4]}$. According to the process of shape setup, the shape self-learning function should learn the data of several sample segments of the strip's head, but the production line has not the shape feedback function, which resulting in strip body and tail in the open-loop state, not guaranting its profile. The setup value of the bending force and the actual bending force are shown in Figure 2. As can be seen from the figure, the bending force of the head is significantly smaller than the value of the body and tail. If the shape self-learning function makes use of the bending force of the strip's head, it will lead to learn the crown learned is greater than the crown of the full strip, which reducing the accuracy of the basis of the learning and leading to self-learning disorders.

\section{Development of Profile Self-learning}

Considered the deviation between measured value and target value of profile, the profile self-learning model obtains the profile correction of each stand, which is used for the profile setup of next strip. Figure 3 shows the strategy of the profile self-learning model. 


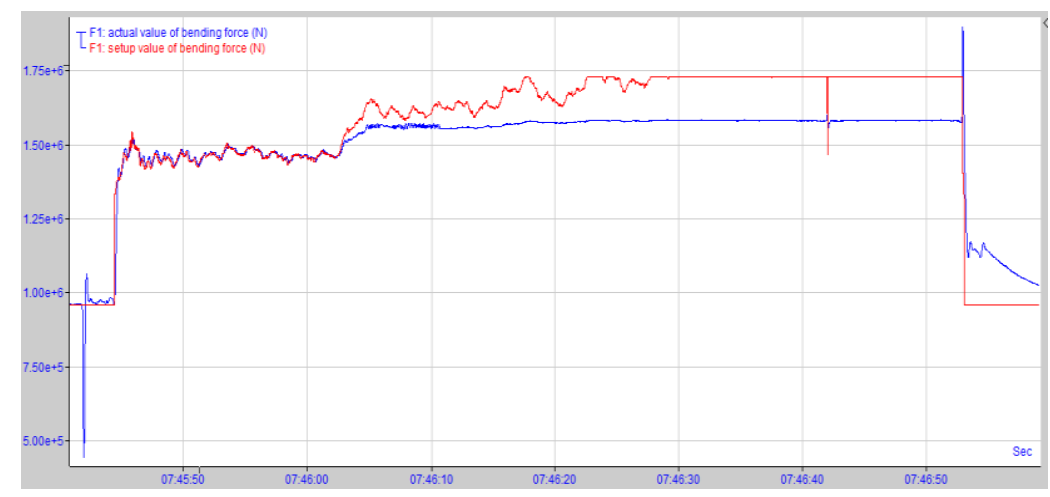

Fig. 2: The Actual and Setup Bending Force

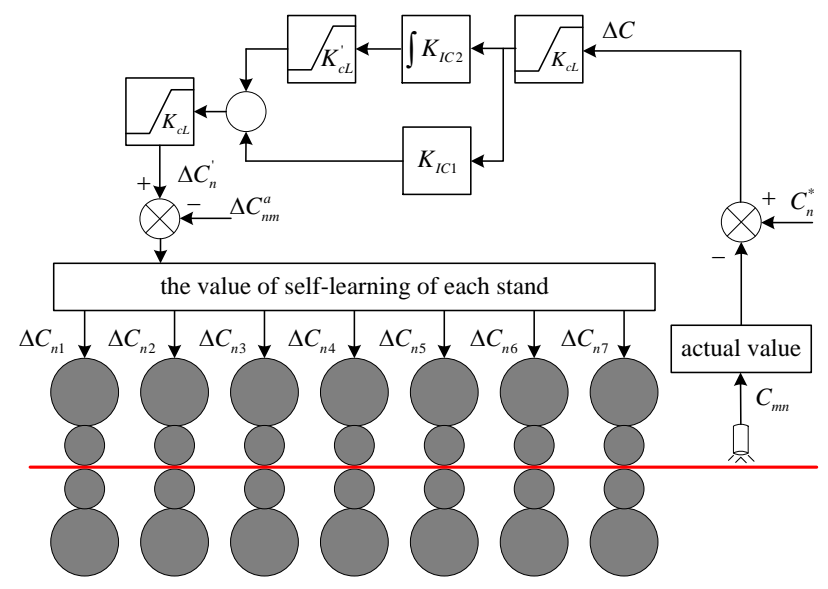

Fig. 3: The Strategy of The Shape Self-learning Model

The profile self-learning strategy is shown as follows:

(1) Calculate the deviation:

$\Delta C_{n}=C_{n}^{*}-C_{m n}$

$C_{n}^{*}$ - Target profile;

$C_{m n}-$ Measured profile;

(2) Calculate the profile deviation after the controller: $\Delta C_{n}^{\prime}$;

(3) Calculate the value which is assigned to each stand:

$\Delta C_{n i}=c_{a i} \cdot\left[\frac{\Delta C_{L i}}{\Delta C_{L}^{T}}\right] \cdot\left[\Delta C_{n}^{\prime}-\Delta C_{n m}^{a}\right]$

$\Delta C_{n i}$ - Target profile of stand $i$

$c_{a i}$ - Weighting factor of stand $i$;

$\Delta C_{L i}$ - Limit value of profile adjustment;

$\Delta C_{L}^{T}$ - Total limit value of profile adjustment;

$\Delta C_{n m}^{a}$-Target profile deviation;

In order to truly reproduce the actual rolling process and maintain the consistency of the setup model with the self-learning model, the self-learning peocess is as follows: 


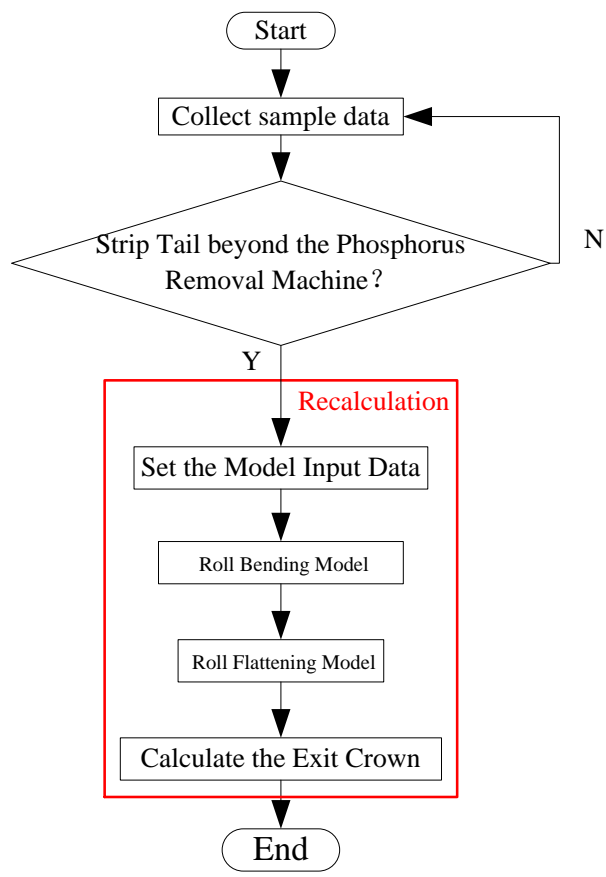

Fig. 4: The Flow Chart of Recalculation

\section{Application}

The new model has been applied, and the deviation between the shape setup calculation and the shape self-learning calculation is getting smaller. The hit rate of the profile increased by about $2 \%$ and the self-learning speed is getting faster. The data statistics results are shown in Figure 5.

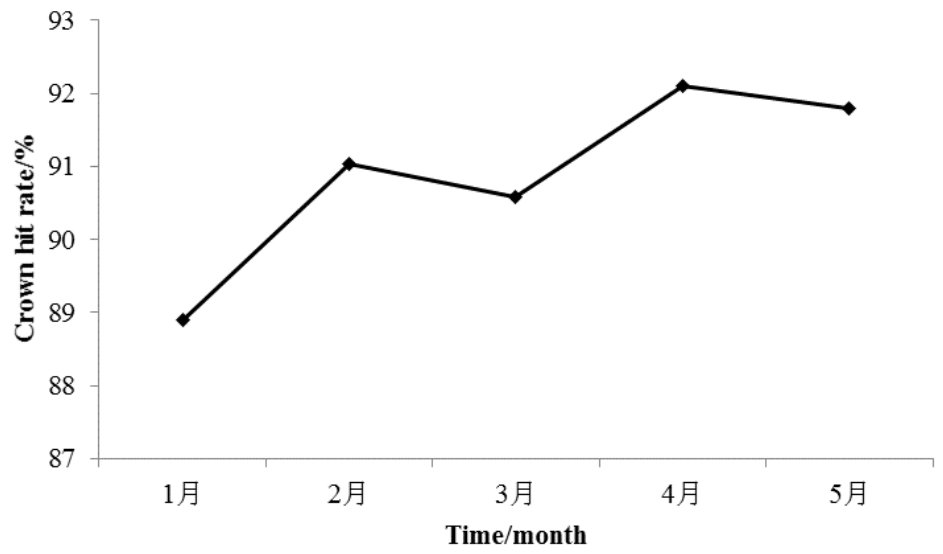

Fig. 5: Application Effect

\section{Conclusion}

Based on study of the profile control model for HSM, a new profile self-learning model has been developed. The new model avoids the lack of no level-I profile control feedback function, by increasing the sampling data. The practical application shows that the new algorithm improves the convergence speed of the profile self-learning and the production efficiency. Although the new function has great improvement for profile, but there are still some problems which should be studied deeply. 


\section{References}

[1] Chen Xian lin, Zou Jiaxiang, A Specialized Finite Element Model for Investigating Controlling Factors Affecting Behavior of Roll and Strip Flatness, 4th International Steel Rolling Conference. Deauville: 1987.

[2] Bald W, Beisemann G, Feldmann H, et al. Continuously Variable Crown Rolling, Iron and Steel Engineer, 1997, 64(3).

[3] Yikang Sun, Model and Control of Cold and Hot Strip Mill, 2010, pp. 205-239.

[4] Xiangqun, Weigang Li, et, Research and Application of Optimizing Setting Strategy of Bending Roll Force in Hot Continuous Rolling, Journal of Wuhan University of Science and Technology, 2016, 39(6). 